Technological University Dublin ARROW@TU Dublin

2003

\title{
Agent Chameleons: Agent Minds and Bodies
}

\author{
Brian Duffy \\ Media Lab Europe \\ Gregory O'Hare \\ University College Dublin \\ Alan Martin \\ University College Dublin
}

See next page for additional authors

Follow this and additional works at: https://arrow.tudublin.ie/scschcomcon

Part of the Computer Sciences Commons

\section{Recommended Citation}

Duffy, B., O'Hare, G., \& Martin, A. (2003). Agent Chameleons: Agent Minds and Bodies. Proceedings of the 16th International Conference on Computer Animation and Social Agents (CASAí03), 2003., pp.218-225. doi:10.1109/CASA.2003.1199312

This Conference Paper is brought to you for free and open access by the School of Computer Sciences at ARROW@TU Dublin. It has been accepted for inclusion in Conference papers by an authorized administrator of ARROW@TU Dublin. For more information, please contact arrow.admin@tudublin.ie, aisling.coyne@tudublin.ie,gerard.connolly@tudublin.ie.

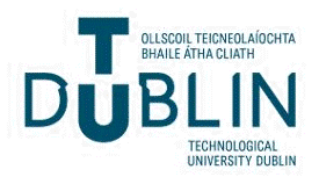




\section{Authors}

Brian Duffy, Gregory O'Hare, Alan Martin, John Bradley, and Bianca Schoen-Phelan 


\title{
Agent Chameleons: Agent Minds and Bodies
}

\author{
Brian R. Duffy ${ }^{2}$, Gregory M.P. O’Hare ${ }^{1}$, Alan N. Martin ${ }^{1}$, John F. Bradley ${ }^{1}$, and Bianca Schön ${ }^{1}$ \\ ${ }^{I}$ Department of Computer Science, University College Dublin (UCD), Belfield, Dublin 4, Ireland \\ ${ }^{2}$ Media Lab Europe (MLE), Sugar House Lane, Crane Street, Dublin 8, Ireland \\ E-mail: \{gregory.ohare, alan.martin, john.bradley\}@ucd.ie, \{brd, bianca\}@mle.ie
}

\begin{abstract}
Agent design has to date concerned itself with the issues pertaining to a single body embedded in a single environment, whether virtual or real. This paper discusses the notion of an agent capable of migrating between information spaces (physical worlds, virtual reality, and digital information spaces). An architecture is presented that facilitates agent migration and mutation within such environments. This will in turn support agent evolution the ultimate in agent adaptivity.
\end{abstract}

\section{Introduction}

The Agent Chameleon Project strives to develop digital minds that can seamlessly migrate, mutate and evolve on their journey between and within physical and digital information spaces. This challenges the traditional boundaries between the physical and the virtual through the empowerment of mobile agents. Three key attributes mutation, migration and evolution underpin the Agent Chameleon concept.

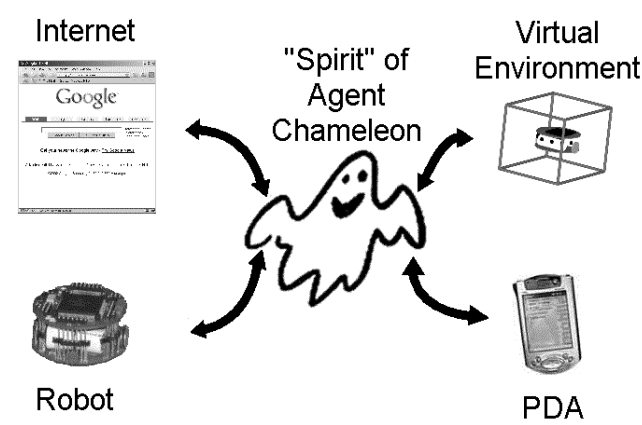

Figure 1. The Agent Chameleon spirit

The ultimate survival and longevity of agents is predicated by their ability to sense, react and respond to environmental change. The response can take the form of migration across a wireless network, mutation of agent form, or evolution of the agents' form and associated capabilities. The form of an agent inextricably dictates or constrains its behaviour and capabilities within a particular environment. The optimum form is very much dependent upon its world [21]. Judicious selection of appropriate forms or persona ought to empower the entity.

Within this paper an architecture and agent structure is described which supports seamless migration and mutation across platforms and within environments. Such agent adaptivity and mobility has thus far not been investigated in the literature.

\section{Related Research}

The Agent Chameleons project draws on a number of major bodies of research and seeks to extend current interpretations of agent systems, virtual environments, and embodied systems (robotics). This work builds upon seminal work conducted by the Collaborative Virtual Environment (CVE) community. Notable pioneering systems that incorporate agent-based techniques include DIVA (Distributed Intelligent Virtual Agent) [32], MAVE (Multi-agent Architecture for Virtual Environments) [7], STEVE [15], Trilogy [20], AgentSalon [30], and ECHOES [22][26].

The realisation of evolvable characters in virtual environments will draw inspiration from such work as Synthetic Characters at MIT-Media Laboratory [2], and work on agents as synthetic characters [1][5][11][14][27].

Although the principles of mobile agents have started to develop (i.e. [8] mIVA [19], m-P@gents [31]), few have embraced a true sense of mobility of an agent through information spaces. The term mobile agent has incorrectly referred to data flow between agent mechanisms, mobile components of a static agent, and notions of data inheritance of one agent from another. The following sections discuss the fundamental issues that arise when the agent becomes truly mobile.

\subsection{Environment \& Embodiment}

Terminology and its misuse continuously create confusion. This section reflects upon the terms situatedness, embodiment, and immersion and the interplay between these terms in order to set the 
foundations for subsequently presenting work on agent migration and mutation.

The Agent Chameleon project [23] [25] explicitly adds an increased degree of complexity to the issue of context and thereby necessitates a more distinct clarification of terminology. When an agent has the facility to migrate between real and virtual information spaces, the issue of embodiment, immersion and situatedness becomes unclear. To illustrate, the virtual representation of an agent in virtual reality in the form of an avatar and controlled through such devices as data-gloves is often referred to as immersion of the user in VR. Similarly, when this agent migrates to a hardware platform, the primary context for actuation and sensing becomes the physical world, which is often referred to as physical embodiment. The following represents common interpretations that have been employed in (and often transferred between) research fields dealing with the idea of realising a computational system of some form.

Situatedness: A software agent in a software environment and the extent to which the agent is situated in this environment [18]. Brooks' interpretation of situatedness is based primarily on reactive behaviour without environmental representation [3].

Embodiment: The physical and social manifestation of a robot in our physical and social world [13][28]. It refers to the provision of a tangible physical body and the design and development of a control architecture such that "robots have bodies and experience the world directlytheir actions are part of a dynamic with the world and have immediate feedback on their own sensations" [4].

Immersion: The manifestation of a virtual character (avatar) in a virtual environment, often in the form of a virtual persona of a real person [32] [20], [22]. Immersion is the sense of displaced embodiment where the sense of being immersed in a Virtual Reality space is facilitated through interactive modalities such as data gloves, motion tracking and position sensing.

Context is the all-encompassing term that is instantiated as situatedness, embodiment and immersion in different fields of research. Context constitutes a metalevel concept, which associates the actions and perceptions of a system with its environment. The context is viewed as a triple of task or activity, the "body" and the environment. The idea of context in artificial systems now has a new dimension. In this work, the specifics of the context for the Agent Chameleon equipped with the ability to migrate between different environments, changes. It can be immersed in VR, embodied in a robot, or situated on a PC or PDA accessing the Internet or databases. In order to do this, the traditional issues regarding mind and body in $\mathrm{AI}$ come to the fore.

Agent Chameleons transcends the often-misused notion of embodiment in AI by emphasising the more appropriate/reflective issue of embodiment: complete adaptivity. A strong sense of intelligence requires a strong sense of embodiment, or complete embodiment. Not only does embodiment require being situated, which can be superficially or easily dealt with by providing a body or embodying an agent in the physical world and effectively requiring it to interact with the physicalities, but it also includes a sense of immersion. The Agent Chameleons project takes an alternative slant on immersion: a stronger sense of context and adaptability as realised in a seamless integration across virtual reality and the actual physical reality. That is, the agent is so immersed in the context that both physical and virtual worlds merge.

\subsection{Mind \& Body}

To use analogies to more clearly illustrate the concepts presented here, the mobile agent can be viewed as an artificial mind with the capacity to change its form by possessing different bodies in different information spaces (i.e. robot in physical space, avatar in VR space). This technology provides for a very interesting turn in the arguments dealing with the development of an intelligent entity and the requirements for strong embodiment in physical reality [28][3][29][17][6][13]. One of the primary criteria for the realisation of an intelligent entity is the integration of the context into the design and implementation of the controlling architecture of the entity. Classical AI begged to differ. Descartes, in Meditations [10], aimed to show that mind is distinct from body in his study of the human body as a machine.

The two perspectives mentioned previously, namely the dualist approach where mind is distinct from body and the embodied approach where mind and body aim to function as one, have aimed with moderate degrees of success to bridge the gap between designed and realised behaviour. Collectively, these approaches are key to the development of the Agent Chameleon. While this can be viewed as a dichotomy, the provision of context for the agent mind, which has the capacity to migrate between bodies, must be implemented in order to achieve the successful realisation of an Agent Chameleon.

Agents that can migrate and mutate their embodied form present significant research opportunities, namely (a) the digital space can become more embedded in our own space and vice versa, (b) the agent can overcome the traditional shortcomings of being constrained to a particular information space, and (c) the classical interpretations of real-world attributes superimposed on an artefact such as physical geometry and constraints (gravity) become less pertinent in VR worlds.

\section{The Agent Chameleons Architecture}

The Agent Chameleons project extends the traditional notions of an agent environment and its constraints by 
expanding through mobility/migration and mutation to virtual environments (i.e. avatar), physical environments (i.e. robot), and software environments (i.e. OS desktops, PDA's) (see figure 2). This capacity to change the context of the agent's actions as it migrates necessitates a new approach to the traditional interpretations of how the environment affects the reasoning mechanisms of the agent.

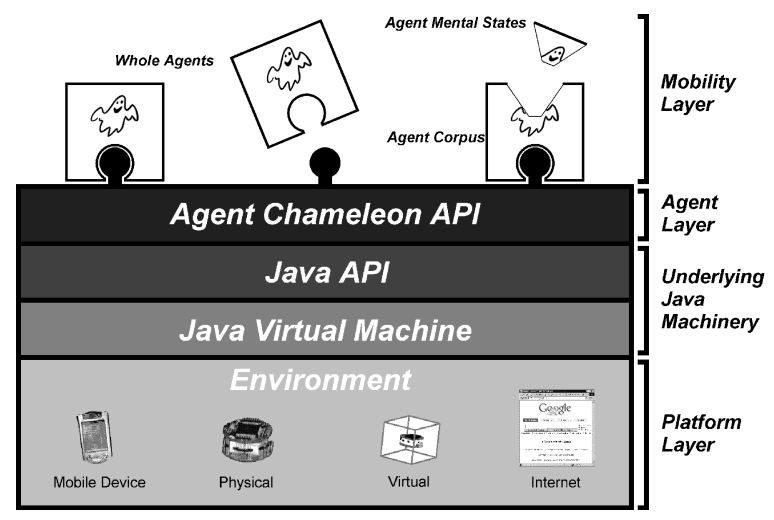

Figure 2. Agent Chameleon Architectural Strata

The Agent Chameleon Architecture developed in the following sections illustrates how this can be dealt with.

\subsection{Agent Architecture}

The architecture of the agents is based upon the Social Robot Architecture (SRA), work carried out by one of the researchers [13]. Like the SRA, "a modular structure is used to divide the levels of complexity into incremental functionality ... More abstract levels provide increasing complexity and subsume lower level functionality. Reactive or reflex survival behaviours are implemented at the reactive level with more complex behaviours defined within the deliberative level" [13]. This architecture is illustrated in Figure 3.

The agent architecture is comprised of three layers Environmental, Reactive and Deliberative. The modular design facilitates reflex behaviours for unexpected or dangerous events that require immediate reaction. Any planning would impede any real-time response required for these reactive behaviours. Hence, there is a higher layer to deal with more deliberative requirements.

The Environmental Layer is responsible for the agent's low-level interaction with its environment. It consists of two types of structures - Perceptors and Actuators.- and each further decomposed into social and platform. Perceptors are responsible for the monitoring of the environment. They pass relevant information about it to the Reactive and Deliberative layers. On the other hand, Actuators are used to affect the environment and are triggered by information from the Reactive and Deliberative Layers.

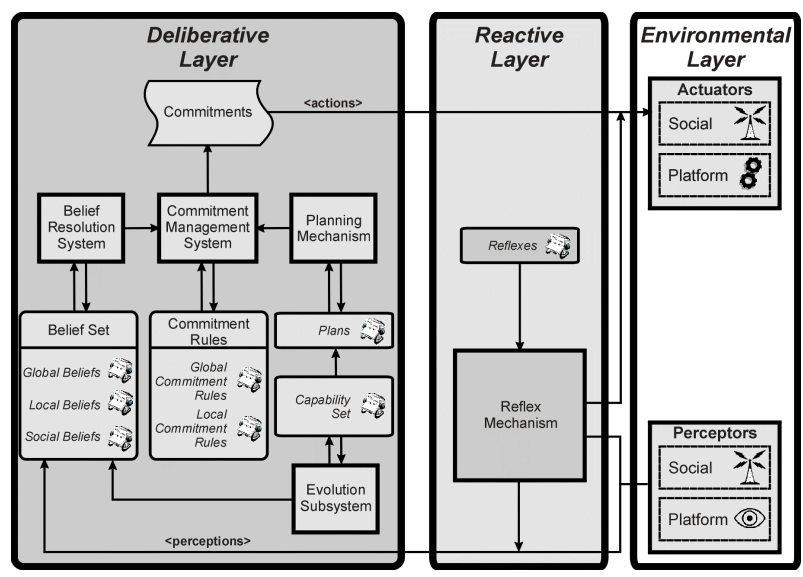

Figure 3. Agent Chameleon Architecture

The purpose of the Reactive Layer is to eliminate reliance on deliberation mechanisms for all agent actions, a classical failing of purely deliberative architectures. A series of basic reflexes empower the agent with a collection of survival instincts. Generally, these behaviours are dependent on the agents environment, e.g. if the agent was in control of the physical robot it would have collision avoidance behaviours. However, there are some behaviours that would be independent of the platform, e.g. if the agent environment became unstable it would attempt to protect itself through migration. After the Reactive Layer performs an action it informs the Deliberative Layer accordingly. In the event that the Reactive Layer is unable to deal with a situation, the problem is passed on as a priority to the deliberative mechanisms.

The Deliberative level provides the necessary functionality to deal with complex tasks that the agent will be required to perform. In order to achieve deliberative proactive agents we use the Belief-DesireIntention (BDI) methodology. Agents are equipped with beliefs about their environment; such as what type of environment it is (e.g. robot, virtual environment, PDA, internet) and what the agent can achieve within this environment. In addition agents are equipped with beliefs about other environments, what constraints are in those other environments and whether they are capable of migration to those environments. A series of commitment rules help to drive the agents towards their goals. The mechanisms employed to maintain consistency across platform migration are based on a functionality set with active and inactive components depending on the instantiation. This facilitates the knowledge set of the agent in choosing possible body instantiations for particular problem sets. 
The deliberation mechanisms are based upon Agent Factory (AF) [8][24], an agent prototyping environment designed and developed at UCD. The architecture provides the agents with the ability to reason based upon beliefs, desires and intentions. The agent structure consists of:

- A Mental State - the agents Belief Set constitutes its mental state. The Belief Set is comprised of Global Beliefs, Local Beliefs and Social Beliefs. The Global Beliefs are comprised of information that is relevant to the agents overall plan and not dependent on platform. The Local Beliefs contain information pertaining to platform dependent matters. The Social Beliefs are developed based on social interaction between the agent and other socially capable entities. These Beliefs are augmented by information from the Environmental and Reactive Layers.

- Commitment Rules - The Commitment Rules are a core part of the Deliberative Level. These form the rules through which the agent will commit to a particular action based on its current Beliefs Set.

- Commitments - Applying Commitment Rules to Beliefs produce Commitments. They are used to trigger specific actions in the Environmental Layer.

\subsubsection{Capabilities.}

Agent Chameleons are considered as an autonomous, mobile and social entity in the classic multi-agent systems sense. The agent has at any given instance a persona and associated with a given persona are a given set of capabilities. Knowledge of particular platforms and their capabilities is contained within the Capability Set. The agent has two types of capabilities - internal capabilities, which are independent of platform, and environmental dependent capabilities, that require the agent to possess a particular platform to avail of them. The agent utilises the various capabilities of different platforms through migration.

\subsubsection{Social Ability.}

Due to the very nature of the agent's capacity to migrate from platform to platform with a view to utilising alternate capabilities, the social functionality is embedded throughout the architecture in the form of social beliefs, social preceptors, and social actuators. This ensures that the agent is not constrained to a particular configuration based on hardware functionality as seen in the SRA (within the SRA a separate Social Layer was useful because of the consistent nature of the robotic platforms). Social ability is here seen as platform dependent and is dealt with through the Environmental Layer. It should be noted that the agent maintains some degree of social ability as communication mechanisms are used to support migration.

\subsection{Migration}

In order for a successful migration to occur, the agent needs a means of transport and a destination device to support it. Agents locate other platforms through their capabilities sets, which contains a list of other platform's IP addresses, port numbers and their capabilities.

Agent migration is achieved through cloning. When an agent wants to migrate it informs the destination that it wishes to do so. The destination creates an agent. The mental state of the agent is only then copied and transmitted to the required destination. Upon receipt it is incorporated into the new agent. The old agent is then disposed of and the new agent begins execution.

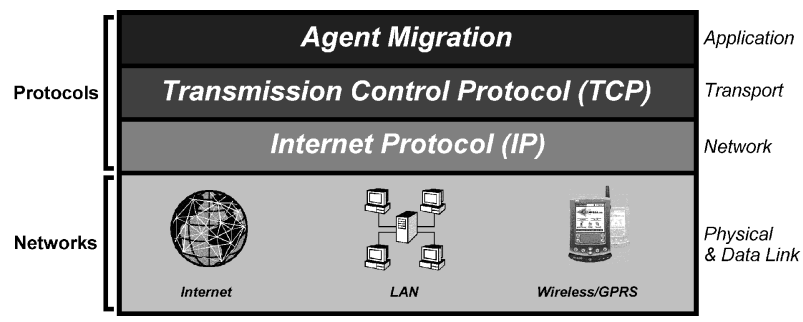

Figure 4. Layered Agent Migration Model

Within the project agent migration protocols are built upon the existing TCP/IP model. This framework was chosen because of its wide-ranging support for data transfer between heterogeneous digital devices.

\subsection{Mutation}

Agent mutation is a core functionality of agent chameleons. The embodied form helps the user and other agents in the recognition and subsequent relationship with the entity. In certain circumstances the guise of an agent may need to change to reflect social context and differing roles. Some persona may not be appropriate within certain environments. For example a fully animated character may prove an appropriate embodied form on a desktop machine, but computationally unfeasible on a PDA. The ability for an agent to self determine its form based upon environmental stimuli offers profound opportunities. It is our conjecture that the agent persona is inextricably linked to their associated capabilities. The mutation thus results in a change to the associated capability set.

As the set of possible agent migration platforms are heterogeneous, issues arise regarding the differing capabilities and restrictions/limitations on these platforms (i.e. limitations in memory, processing power, actuators, preceptors) and how these are dealt with from a control perspective. Agent mutation refers to the agent's capacity to adapt its function and form depending on environmental, platform, and social constraints or freedoms. To illustrate, an agent migrating to a Khepera robot has limited processing capabilities whilst a VR 
agent avatar can have the power provided by a full operating system.

\subsection{Environments}

\subsubsection{The Virtual.}

In order to develop the coherence and fluidity necessary to effectively link the physical and virtual domains, a computational engine as found in computer gaming is used to merge these traditionally distinct environments and facilitate the seamless migration of the digital spirit from one environment to another. This both enhances and facilitates the control of avatars based on either real world or digital-domain sensory information. To develop the virtual environments and avatars, 3D Studio Max is currently used for high-level modelling and animation.

Preliminary proof-of-concept demonstrators were also constructed, using Virtual Reality Modelling Language (VRML). This system builds upon work in the Virtual Robotic Workbench [12], but has been augmented with the Agent Chameleons framework. The VRML scene is updated using the External Authoring Interface (EAI) from within a Java applet.

\subsubsection{The Physical.}

For migration into the physical world, agent chameleons can possess robotic devices, such as K-Teams Khepera robot [16]. The systems in these environs are built upon Connected Limited Device Configuration (CLDC) of the Java 2 Platform Microedition (J2ME). The Khepera robots are embedded with Sun's kilobyte virtual machine (KVM), which is a VM build with constrained devices in mind. The agents utilise TCP/IP protocols to migrate across networks, but light weight robots like the Khepera do not have direct access to a network, so a PC acts as an intermediate relay for communities of these such devices. This gives agents on the Kheperas the ability to communicate/migrate to entities on external networks.

\subsubsection{The Mobile.}

A version of the system has also been created for use on Pocket PC based Personal Data Assistants (PDA's) such as the Compaq iPAQ. This version is based upon the Personal Java compliant Jeode JVM, created by Insignia. This version contains a simpler interface than that of the full VR based one, with agents appearing as 2D animations in a manner akinned to the Microsoft Office Assistant. These icons have a resemblance to the VR characters, so that the presence of the agent is not impeded by the reduced display and processing power of the PDA.

\subsubsection{Data.}

In order for the agent to explore the Internet, a webbrowsing server is provided. This offers a location for the agents to migrate to, from which Internet resources are accessible. Migration to other information sources like a corporate intranet, or a specific database, could similarly be supported.

\section{Experiments}

By way of exercising the architecture described in section 3 we present three simple demonstrators. The first demonstrates agent migration between real and virtual spaces, the second depicts an example of external mutation in response to changing environmental conditions, and the final demonstrator displays an example of the agents survival instincts in response to an unstable environment. In all three examples the agent behaviour of migration, mutation and survival is underpinned by the same decision making process, namely a BDI agent structure. In each of the 3 examples we will thus provide snapshots of the agent mental state. This illustrates how belief adoption drives commitment adoption, which in turn determines agent action.

\subsection{Basic Migration}

This demonstrator illustrates the migration of an agent from a physical, real world, robot to a virtual space and vice-versa. In this experiment, a physical world is extended by a virtual world depicted on a computer screen adjoined to the physical world. Small Khepera robots can navigate and explore the desk-mounted world and dock in a robot garage at the edge of the physical world thus removing the physical robot from vision (see Figure 5). Thereafter the robot seamlessly crosses into the virtual world and a virtual robot continues the trajectory of the physical counterpart into the virtual space.

Figures 5, 6, 7 and 8, indicate the mental states of the agent at various stages of the experiment. Beliefs, commitments and commitment rules extraneous to the experiment have been omitted from the figures for conciseness.

Figure 5 illustrates the pre-migration mental state. Two commitment rules relating to migration are visible. The first indicating if an agent has a desire to migrate it should adopt a commitment to find a garage the second indicating that if the agent is in the garage and wants to migrate then it should adopt a commitment to migrate. Figure 6 depicts the activation of the first commitment rule at a subsequent time point, while Figure 7 shows a subsequent belief update relating to a commitment to migrate. Figure 8 illustrates that the migration has now been effected as evidenced by the robot emerging from the virtual garage and by virtue of the agent believing its network address has now changed from 82 to 77 . 


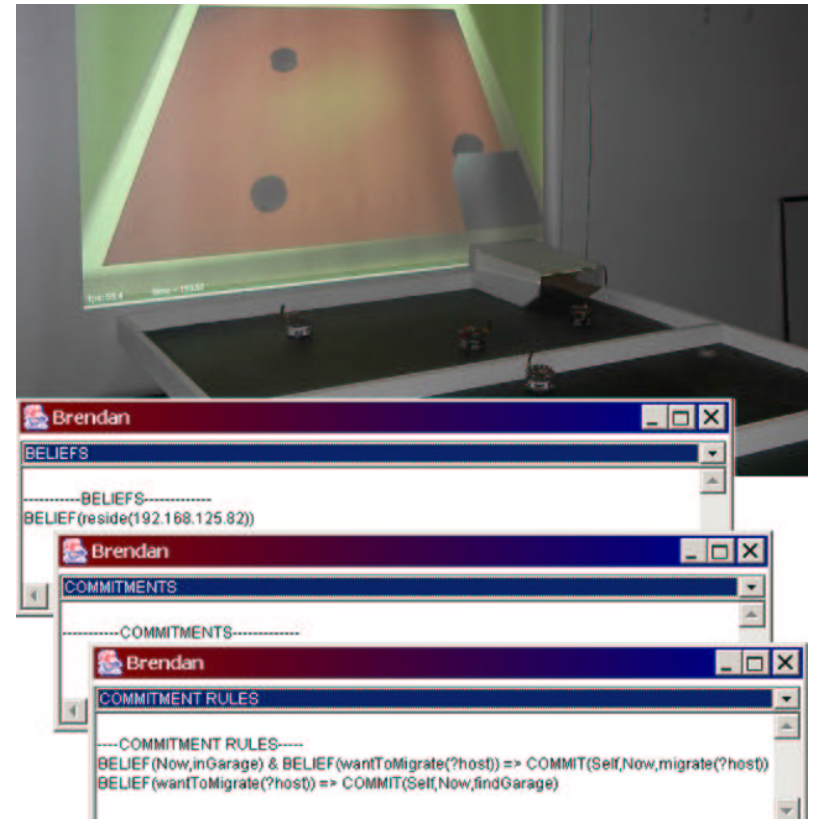

Figure 5. Pre-migration Mental State

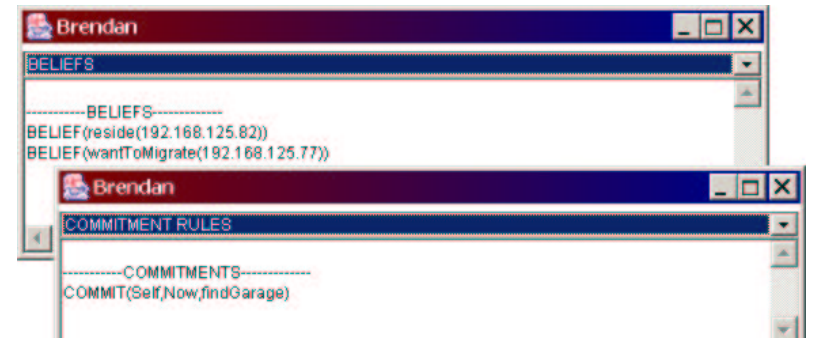

Figure 6. A Desire to Migrate

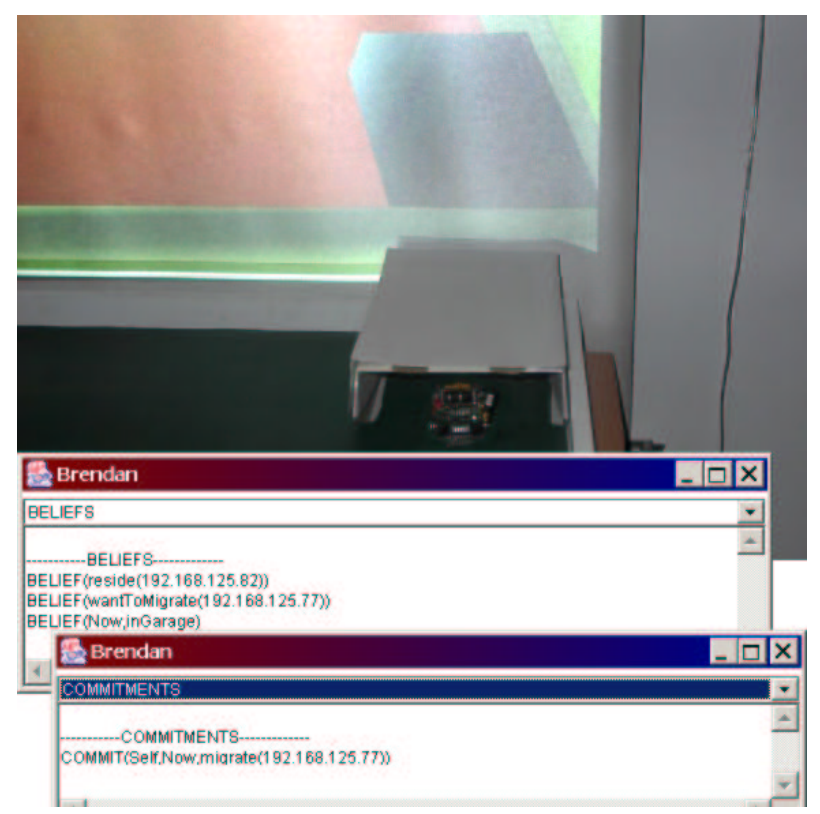

Figure 7. Commitment to Migrate

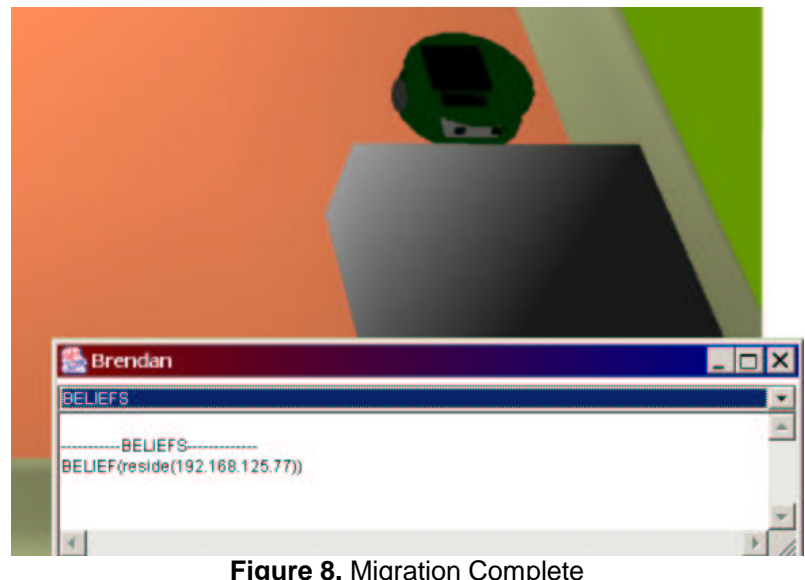

Figure 8. Migration Complete

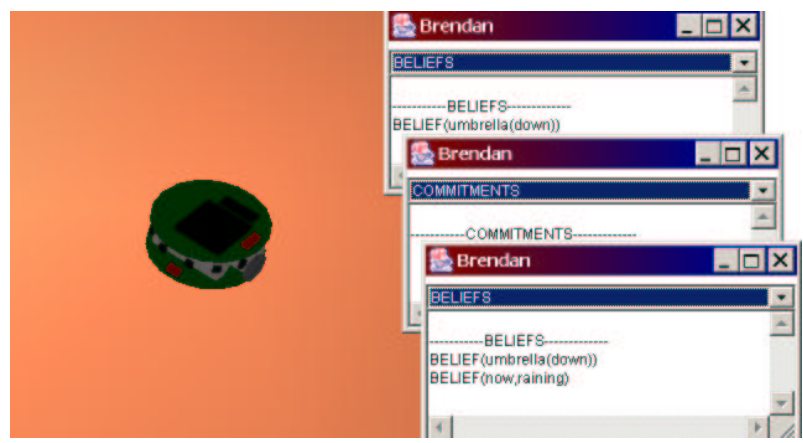

Figure 9. Pre-mutation Mental State

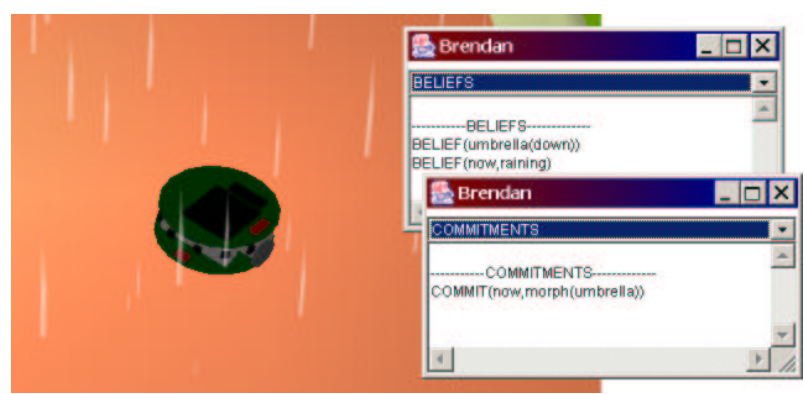

Figure 10. Commitment to Mutate

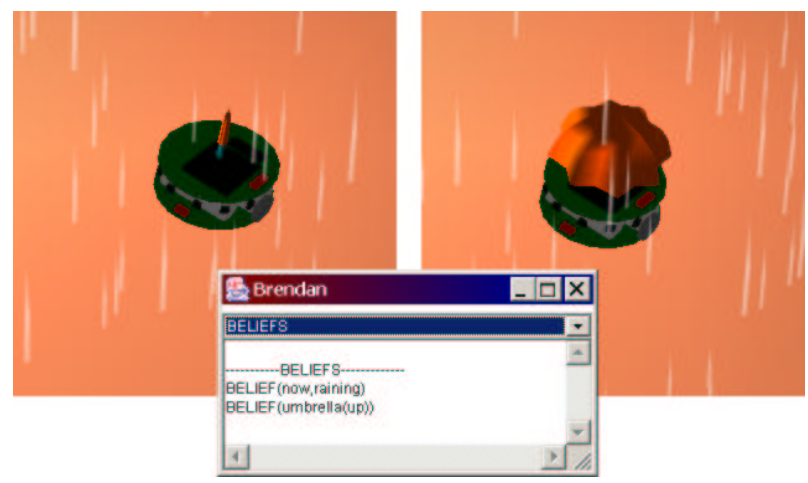

Figure 11. Post-mutation Mental State 


\subsection{External Mutation}

To date various mutations of the virtual agent have been realised. Such mutations are underpinned by commitment rule invocation. For example, in response to a change in the environmental conditions, such as a change in the weather within the virtual environment, the agents may mutate their form. This is illustrated in Figures 10 and 11 . When the situation changes in the environment, i.e. when it begins to rain, a belief is generated by a perceptor. Based upon the commitment rules the agent commits to raising the umbrella, triggering an actuator that actually changes the form.

In a similar manner Figures 9, 10 and 11 illustrate the pre-mutation, mutate adoption and post migration mental states. While the mutation contained within this example constitutes little more than morphing an avatar, mutation is generally much more complex and results in the change of the external or embodied form and the associated capabilities. The capabilities of an agent are inextricably related to the agent form.

\subsection{Survival}

The agents used in this research have been attributed basic survival instincts based on the ability of their environment to support their continued operation. For example an agent would have a perceptor monitoring the power supply to its current environment.

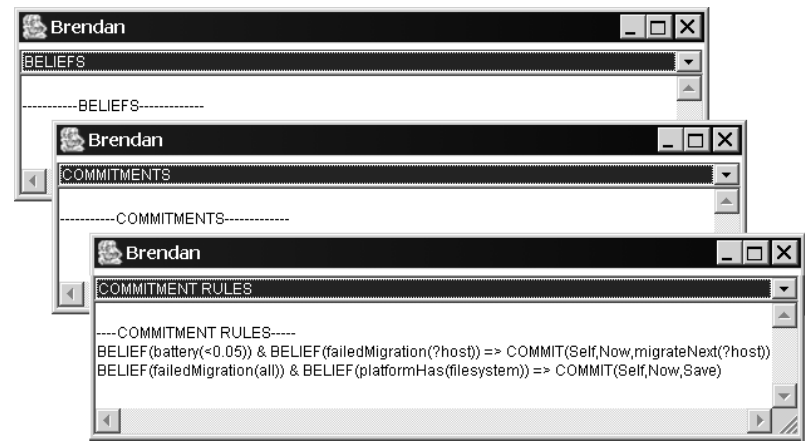

Figure 12. Pre-survival Mental State

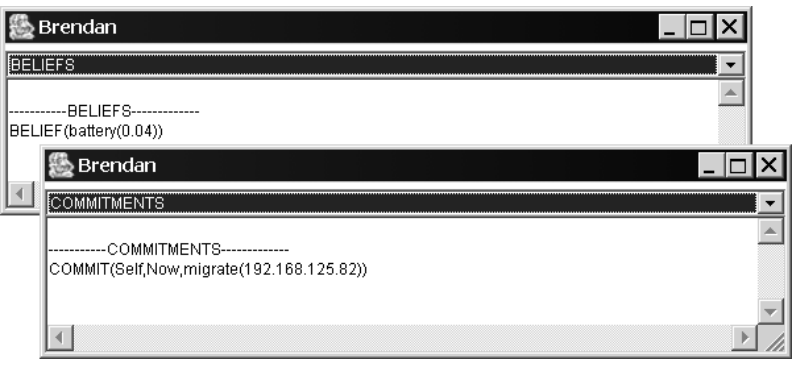

Figure 13. Survival Mental State
The Figures 12 and 13 illustrate the changes in mental state in the event of diminished power. This is of course a very rudimentary survival behaviour and other much more sophisticated behaviours are possible in hostile or competitive environments.

\section{Conclusion}

Within this paper we have introduced the concept of Agent Chameleons. Such deductive entities reside within embodied containers and exhibit the key attributes of autonomy, mobility, mutation and an ability to evolve. We regard mutation and evolution as higher order attributes synonymous with chameleon agents a new and more sophisticated agent class.

The Agent Chameleons Architecture provides the necessary computational support for such migration and mutation. In such nomadic agent environments the degree of social cohesion is typically weaker where agent dynamics produce primarily transient relationships. This is further compounded by the mutation of agent forms, which degrades the visual cues that assist recognition and relationship formation. On-going work is examining the derivation of models of trust reliance and dependence within such nomadic agent communities.

We have provided a brief insight into three proof of concept demonstrators that illustrates the fact that mutation and migration are underpinned with the same base BDI architecture. Similar to other actions commitments to mutate and migrate are adopted and actuators subsequently realise these actions.

Acknowledgements. The work undertaken as part of the Agent Chameleons project (http://chameleon.ucd.ie) is a collaborative project between the Department of Computer Science, University College Dublin (UCD) and Media Lab Europe (MLE), Dublin. We gratefully acknowledge the financial support of the Higher Education Authority (HEA) Ireland.

\section{References}

[1] N.I. Badler, C.B. Phillips, and B.L. Webber, Simulating Humans: Computer Graphics, Animation, and Control, Oxford University Press, 1993.

[2] B.M. Blumberg, Old Tricks, New Dogs: Ethology and Interactive Creatures, Ph.D Dissertation, MIT, 1996.

[3] R.A. Brooks, "A Robust Layered Control System for a Mobile Robot”, IEEE Jour. Rob. and Autom., 2(1) 1986

[4] R.A. Brooks, Cambrian Intelligence, Cambridge, MA, MIT Press, pp. 138-139

[5] J. Cassell, C. Pelachaud, N. Badler, M. Steedman, B. Achorn, T. Becket, B. Douvillle, S. Prevost, and M. Stone, "Animated Conversation: Rule-based Generation of Facial 
Expressions, Jesture \& Spoken Intonation for Multiple Conversational Agents", In Proceedings of SIGGAPH '94, 1994.

[6] A. Clark, Being There: Putting Brain, Body, and World Together Again, MIT Press. 1997.

[7] J. Cobel, and K. Harbison, "MAVE: A multi-agent architecture for virtual environments", in Proc 11th Int. Conf. on Industrial and Engineering Applications of artificial intelligence and Expert Systems, LNAI, Vol.1415, Springer Verlag, 1998.

[8] R.W. Collier, Agent Factory: An Environment for Prototyping Agents. PhD Thesis, Department of Computer Science, University College Dublin, Dublin, Ireland, 2001.

[9] R.W. Collier, C.F.B. Rooney, R.P.S. O’Donoghue, G.M.P. O'Hare. "Mobile BDI Agents" 11th Irish Conference on Artificial Intelligence \& Cognitive Science, University College Galway, Ireland, 2000.

[10] R. Descartes, Discourse on Method and Meditations on First Philosophy, Indianapolis/Cambridge Hackett Publishing 1993, 3rd edition.

[11] P. Doyle, and B. Hayes-Roth, "Agents in Annotated Worlds", In Proceedings of 2nd international Conference on Autonomous Agents, Minneapolis, MN, USA, ACM Press 1998.

[12] B.R. Duffy, G.M.P. O'Hare, R.P.S. O'Donoghue, C.F.B. Rooney, R.W Collier. "Reality and virtual reality in mobile robotics", Managing Interactions in Smart Environments (MANSE'99), Nixon, P., Lacey, G., Dobson, S., Springer Verlag, ISBN 1-85233-228-X

[13] B.R.Duffy, The Social Robot, PhD Thesis, Department of Computer Science, University College Dublin (UCD), Dublin, Ireland, 2000.

[14] L. Foner, "Entertaining Agents: A Sociological Case Study", In Proceedings of 1st international Conference on Autonomous Agents, ACM Press, Marina del Rey, CA, USA, 1997. p122-129.

[15] L. Johnson, and J. Rickel, "Virtual humans for team training in VR", Proc. 9th World Conference on AI in Education, July 1999, pp578-585.

[16] K-Team. Khepera. http://www.k-team.com

[17] G. Lakoff, Women, Fire, and Dangerous Things, University of Chicago Press, 1987.

[18] C. Lueg, and R. Pfeifer, "Cognition, Situatedness, and Situated Design," Proc. 2nd Int'l Conf. on Cognitive Technology Aizu, Japan, August 25 - 28, 1997

[19] P. Pérez, G. Méndez and A. Antonio. "mIVA: Why to Use Mobile Agents in Virtual Environments and Wireless Devices", ECAI 2002, 15th European Conference on Artificial Intelligence. Workshop W6, Artificial Intelligence in Mobile System, Lyon, France. July 2002.

[20] T.J. Norman, N.R. Jennings, "Constructing a virtual training laboratory using intelligent agents", Int Journal of Continuous Engineering and Life-Long Learning, 2000.
[21] G.M.P. O'Hare. "Agents, Mobility and Virtuality: A Necessary Synergy", In Proceedings of International ICIS Symposium on Multi-Agents and Mobile Agents in Virtual Organizations and E-Commerce (MAMA '2000), December 11-13, 2000, Wollongong, ICSC Academic Press, ISBN 3-906454-24-X, 2000.

[22] G.M.P. O'Hare, K. Sewell, A. Murphy, and T. Delahunty, "ECHOES: An Immersive Training Experience", In Proceedings of International Conference on Adaptive Hypermedia and Adaptive Web-based Systems (AH2000), Springer Verlag.

[23] G.M.P. O'Hare, B.R. Duffy, "Agent Chameleons: Migration and Mutation within and between Real and Virtual Spaces", The Society for the Study of Artificial Intelligence and the Simulation of Behaviour AISB 2002, Imperial College, England, April 3-5, 2002.

[24] G.M.P. O'Hare, P.T. O'Hare, and T.D. Lowen, "Far and A WAY: Context Sensitive Service Delivery through Mobile Lightweight PDA hosted Agents", Proceedings of 15th International Florida Artificial Intelligence (FLAIRS) Conference. AAAI Press. Pensacola, Florida. May 14th16th, 2002

[25] G.M.P. O'Hare, B.R. Duffy, J.F. Bradley, and A.N. Martin, "Agent Chameleons: Moving Minds from Robots to Digital Information Spaces", Proceedings of Autonomous Minirobots for Research and Edutainment (AMiRE) 2003 Brisbane, Australia, February 18-21 2003.

[26] A. Pasquarelli, F. de Stefani, G.M.P. O'Hare, \& A.J. Murphy, "ECHOES: EduCational Hypermedia On-linE System", Proceedings of IEEE MultiMedia Conference, Firenze, Italy June 19th-21st 1999.

[27] W.S. Reilly. Believable Social and Emotional Agents, PhD dissertation, CMU 1996.

[28] N. Sharkey, and T. Zeimke, "A consideration of the biological and psychological foundations of autonomous robotics", Connection Science, 10, P361-391, 1998.

[29] L. Steels, "Building Agents with Autonomous Behaviour Systems", The 'artificial life' route to 'artificial intelligence'. Building situated embodied agents. Lawrence Erlbaum Associates, New Haven. 1994

[30] Sumi, Y., Mase, K., "AgentSalon: Facilitating Face toFace Knowledge Exchange through Conversations Among Personal Agents", Fifth International Conference on Autonomous Agents (Agents 2001), pp.393

[31] K. Takashio, M. Mori and H. Tokuda. "m-P@gent: A Framework of Environment-Aware Mobile Applications", Proc. of IEEE International Workshop on Networked Appliances, IWNA 4, 2002.

[32] S. Vosinakis, G. Anastassakis, and T. Panayiotopoulos, "DIVA: Distributed Intelligent Virtual Agents", Proc. VA99, Workshop on Intelligent Virtual Agents, at UK, VRSIG, Sept 1999. 